\title{
THE LUMINOSITY FUNCTION OF THE MAIN SEQUENCE STARS IN THE SOLAR NEIGHBORHOOD
}

\author{
YANG TING-GAO \\ Haanxi Astronomical Observatory, Academia Sinica, Shaanxi 710060 \\ SHU CHENG-GANG, FU CHENG-QI AND JIANG DONG-RONG \\ Shanghai Astronomical Observatory, Academia Sinica, Shanghai 200030 \\ AND \\ PENG QIU-HE \\ Department of Astronomy, Nanjing University, Nanjing 210093
}

The Third Catalogue of Nearby Stars (CNS3) was analyzed. A study of the stellar luminosity function in the solar neighborhood with CNS3 was described. The luminosity function for main sequence stars derived from CNS3 was compared with that from CNS2 and that based on the method of photometric parallaxes. The results from CNS3 for stars with $M_{v}<15.5$ were well defined. Luminosity functions for the giants and A, F, G, K and M type main sequence stars are also given, based on CNS3. 